\title{
Science matters
}

\section{Cell signalling in pulmonary vascular cells: do not shoot the messenger!}

\author{
P H Scott, A J Peacock
}

\begin{abstract}
Pulmonary hypertension is associated with pulmonary vascular remodelling, rendering the vessels unresponsive to vasodilators. An understanding of the mechanisms which cause this remodelling is required, which is likely to be linked to changes in vascular tone whether stimulated by hypoxia or other factors. One way to approach this is to try to understand the intracellular signalling pathways associated with hypoxia in pulmonary artery cells. This understanding could provide opportunities for therapeutic intervention using agents which will interfere with the signalling systems.

(Thorax 1996;51:864-866)
\end{abstract}

Keywords: pulmonary hypertension, cell signalling, vascular remodelling, hypoxia.

Pulmonary hypertension may occur as a primary disease (primary pulmonary hypertension, $\mathrm{PPH}$ ) or as a serious and common sequel to most types of cardiopulmonary disease and, in particular, chronic hypoxic lung disease. In either case prognosis is poor. ' Furthermore, secondary pulmonary hypertension probably contributes significantly to the morbidity of chronic obstructive pulmonary disease because, in the setting of destroyed lung parenchyma, pulmonary vascular haemodynamics may make the difference between a sedentary and an active life style. Previous attempts to reverse secondary pulmonary hypertension with vasodilating drugs have largely been unsuccessful for two important reasons. Firstly, the drugs are non-selective resulting in systemic hypotension and, secondly, the histological changes in pulmonary vessels which accompany pulmonary hypertension (pulmonary vascular remodelling) render the vessels resistant to vasodilators. Clearly we need to understand pulmonary vascular remodelling if we are to improve pulmonary haemodynamics in this setting.

The primary stimulus for remodelling is unknown but may be hypoxia or other factors such as stretching of the pulmonary vessels. Different causes may result in slight differences in the vascular morphology but hypoxia remains a useful model for understanding pulmonary vascular control and vascular remodelling. In the hilar vessels hypoxia causes an eight-fold increase in adventitial fibroblasts before there is much change in the endothelial or smooth muscle cell components of the vessel. ${ }^{2}$ In peripheral vessels the dominant effect is on medial smooth muscle cells. This suggests that the process of pulmonary vascular remodelling differs between proximal and peripheral vessels, a fact that may be of fundamental importance.

In both proximal and peripheral remodelling local growth factors are likely to be important. We believe that these growth factors have two main characteristics: firstly, that they are also vasoconstrictors - that is, there is a link between cell replication and vascular control ("cell growth-vasomotor coupling") - and, secondly, that they are derived from the endothelial cell which probably receives the primary stimulus, whether it be stretch, sheer force, or hypoxia. The most likely contenders at present include endothelin-1 (ET-1) and platelet-derived growth factor (PDGF). These are probably opposed by the physiological dilators nitric oxide and atrial natriuretic peptide.

Whatever growth promoting factor is postulated, there is likely to be a common mechanism that transduces a stimulus such as hypoxia or stretch into a response such as vascular cell replication. We need to understand this mechanism. The flourishing discipline of cell signalling is likely to provide the key (fig 1). In addition, a greater understanding of these intracellular signalling mechanisms may identify possible target sites for the development of novel treatments for pulmonary hypertension.

\section{Scientific background}

Consistent with the concept of "cell growthvasomotor coupling" is the view that cell signalling events associated with contraction may also regulate cell growth and division of endothelial cells, smooth muscle cells, and adventitial fibroblasts. A vast array of growth factors regulate cell function by interacting with specific receptors located upon the cell membrane. This results in the triggering of a chain of specific intracellular signalling pathways and the formation of a number of key 


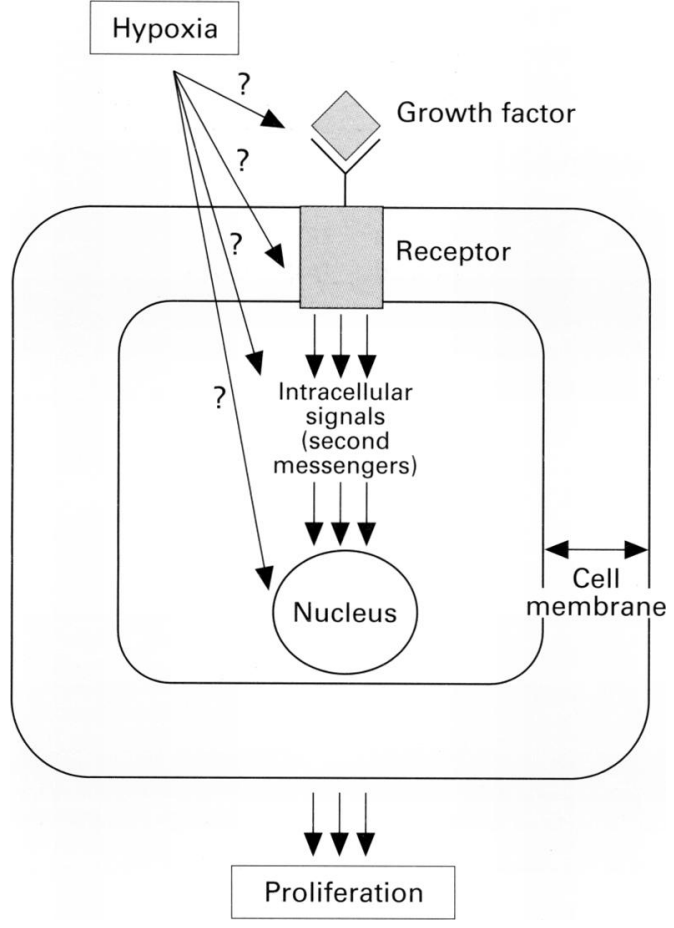

Figure 1 Schematic representation of intracellular signalling pathways leading to cell division. Hypoxia, a stimulator of pulmonary vascular remodelling, could act at several levels in the chain of intracellular signalling events leading to cell division.

second messenger effector molecules (fig 1). We do not yet know which of these signalling events are involved in hypertensive remodelling of the pulmonary artery.

An example of this signalling system is the hydrolysis of inositol-containing phospholipids, found in the cell membrane, and this has been identified as a universal signalling pathway activated in response to agents responsible for calcium mobilisation. ${ }^{3}$ In many cell types this pathway is activated in response to vasoconstrictors such as angiotensin II, ET-1, and $\mathrm{PDGF},{ }^{45}$ but this activation occurs via a different type of receptor. Angiotensin II and ET-1, for example, activate a number of phospholipase effector molecules which are modulated via an intermediate transducing protein, guanosine nucleotide regulatory binding proteins (G proteins), while for PDGF the effector molecules associate with the PDGF receptor itself, become phosphorylated, and are subsequently activated without an intermediary step. Following activation of either receptor type, the phospholipid phosphatidylinositol 4,5bisphosphate is hydrolysed by a phospholipase $C$ (PLC) to generate two second messengers, inositol 1,4,5-trisphosphate $\left(\mathrm{InsP}_{3}\right)$ and diacylglycerol (DAG) (fig 2). Different isoforms of PLC (PLC $\beta$ or PLC $\gamma$ ) are activated at the different receptors but the products of the reaction are similar (fig 2 ). InsP $P_{3}$ stimulates the release of calcium from intracellular calcium $\left(\mathrm{Ca}^{2+}\right)$ stores through the interaction with specific InsP $\mathrm{P}_{3}$ receptors located on the endoplasmic reticulum which has the effect of increasing intracellular $\mathrm{Ca}^{2+}$ levels. The other lipid product of phosphatidylinositol hydrolysis, DAG, is recognised as the main physiological activator of protein kinase C (PKC) which appears to be an important enzyme required in the steps leading to cell division. ${ }^{67}$ In the presence of DAG and calcium ions, activated PKC stimulates the phosphorylation and activation of a number of target proteins which may be involved in proliferation (fig 2).

It is now realised that not all intracellular signals result from the pathway described above. Recent major advances have been achieved in defining cell signalling pathways and these additional pathways involve the activation of proteins or enzymes by phosphorylation upon tyrosine residues. In these pathways, signals are generated through a series of protein/protein interactions and a cascade of phosphorylation events rather than generation of second messengers as in the phosphoinositide pathway described above. This type of pathway has been associated with growth factor receptors that display tyrosine kinase activity such as PDGF or epidermal growth factor; however, a number of $G$ protein coupled pathways can also activate tyrosine kinase pathways.

An example of one of the pathways initiated by different agonists is the activation of a family of intracellular protein kinases known as the mitogen-activated protein kinases (MAP kinases) which have emerged as signalling molecules common to pathways believed to be important in regulation of cell proliferation. ${ }^{9}$

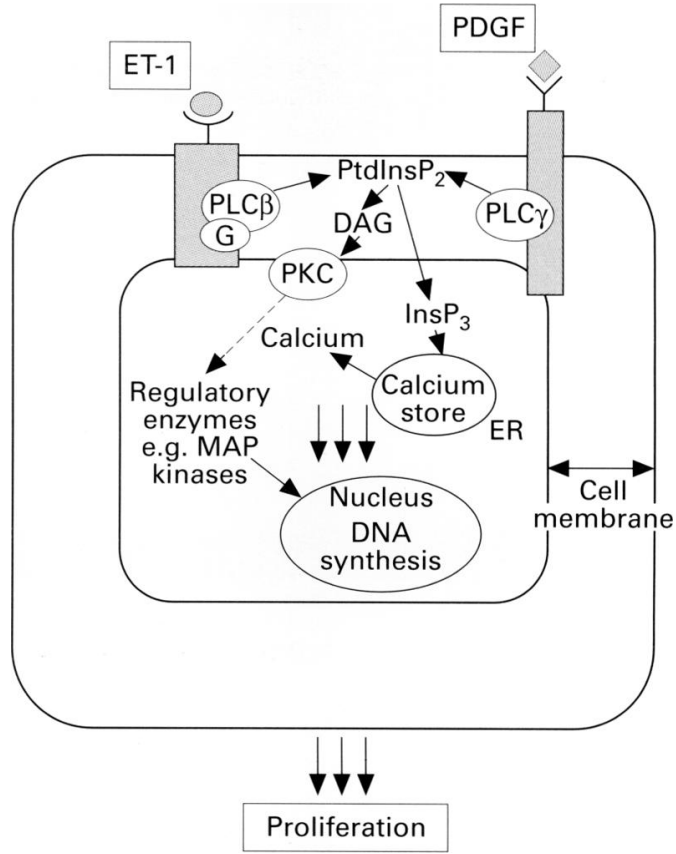

Figure 2 Potential pathways for receptor-mediated second messenger generation and downstream effects leading to DNA synthesis and cell division. Growth factors such as $P D G F$ bind to specific receptors on the cell membrane resulting in association of PLC $\gamma$ to the activated receptor and subsequent hydrolysis of PtdIns $P_{2}$ to form InsP $P_{3}$ and $D A G$. Ins $P_{3}$ binds to specific receptors on endoplasmic reticulum which results in the release of calcium, necessary for cell contraction. DAG activates PKC whose activity is also regulated by calcium levels. Mitogens such as ET-1 can also activate this pathway, however, this receptor activates PLCB. Following activation of regulatory proteins such as $M A P$ kinase, the cell receives a signal for synthesis of new $D N A$ and cell division to occur. DAG = diacylglycerol; $E R$ = endoplasmic reticulum; $E T-1$ = endothelin; $G=G$ protein; Ins $P_{3}=$ inositol trisphosphate; $P A=$ phosphatidic acid $; P D G F=$ platelet - derived growth factor; $P K C=$ acid; $P$ protein kinase $C ; P L C=$ phospholipase $C ; \operatorname{PtdIns} P_{2}=$
phatel $=$ phosphatidylinositol 4,5-bisphosphate. 
Activation of MAP kinases occurs in response to many agonists such as PDGF and ET-1 in a number of different cell types and has been shown to lie downstream from PKC. MAP kinases and other signalling pathways may provide further links in the chain of signalling events leading to cell division. The realisation that various kinases may be important in cell signalling has led to the further identification of specific phosphatases which can inhibit these processes. ${ }^{10} 11$ Future research will concentrate on this balance between phosphatases and kinases.

\section{Therapeutic potential}

Different growth factors activate different signalling events within the cell. Great progress has been made in the understanding of these pathways and it is reasonable to expect that activation of a particular pathway occurs when a vascular cell is stimulated to contract or divide. If we can map this pathway in a particular vascular cell in response to a particular stimulus such as hypoxia, then intervention could prevent the unwanted response. Clearly, this form of intervention at an intracellular level is novel but potentially very exciting - whether in vascular cells of patients with pulmonary hypertension or airway cells of patients with asthma.

\section{Conclusion}

Pulmonary hypertension and pulmonary vascular remodelling are linked and mutually self-sustaining. The key to this link is likely to be found in the processes of cell signalling which occur within pulmonary vascular cells in response to stimuli such as hypoxia. Interruption of the link and prevention of the vicious circle of "cell growth-vasomotor coupling" may be the basis of future therapy.

1 Weitzenblum E, Hirth C, Ducolone A, Mirhom R, Rasaholinjanahary J, Ehhart $M$. Prognostic value of pulmonar artery pressure in chronic obstructive lung disease. Thorax 1981;36:752-8.

2 Meyrick B, Reid L. Hypoxia and incorporation of $3 \mathrm{H}$-thymidine by cells of the rat pulmonary arteries and alveolar wall. Am f Pathol 1979;96:51-70.

3 Berridge MJ, Irvine IF. Inositol phosphates and cell signalling. Nature 1989;341:197-205.

4 MacNulty EE, Plevin R, Wakelam MJO. Stimulation of the hydrolysis of phosphatidylinositol 4,5-bisphosphate and phosphatidylcholine by endothelin, a complete mitogen for Rat-1 fibroblasts. Biochem $\mathcal{F} 1990 ; 272: 761-6$.

5 Cook SJ, Wakelam MJO. Phospholipases $\mathrm{C}$ and D in mitogenic signal transduction. Rev Physiol Biochem Pharmacol 1992;119:14-45.

6 Nishizuka Y. The molecular heterogeneity of protein kinase $\mathrm{C}$ and its implications for cellular regulation. Nature 1988 ; 334:661-5.

7 Hug H, Sarre TF. Protein kinase C isoenzymes: divergence in signal transduction. Biochem $\mathcal{F}$ 1993;291:329-43.

8 Malarkey K, Belham C, Paul A, Graham A, McLees A, Scott $\mathrm{PH}$, Plevin, $\mathrm{R}$. The regulation of signalling pathways by growth factor and G-protein-coupled receptors. Biochem f 1995;309:361-75.

9 Davis RJ. The mitogen activated protein kinase signal transduction pathways. $₹$ Biol Chem 1993;268:14553-6.

10 Matozaki T, Kasuga M. Roles of protein-tyrosine phosphatases in growth factor signalling. Cell Signalling 1996; 8: 13-9.

11 Tainer J, Russell P. Cracking tyrosine phosphatases. Nature 1994;370:506-7. 